\title{
ON SOME MOMENT MAPS AND INDUCED HOPF BUNDLES IN THE QUATERNIONIC PROJECTIVE SPACE
}

\author{
LIVIU ORNEA AND PAOLO PICCINNI
}

\begin{abstract}
We describe a diagram containing the zero sets of the moment maps associated to the diagonal $U(1)$ and $S p(1)$ actions on the quaternionic projective space $\mathbb{H} P^{n}$. These sets are related both to focal sets of submanifolds and to Sasakian-Einstein structures on induced Hopf bundles. As an application, we construct a complex structure on the Stiefel manifolds $V_{2}\left(\mathbb{C}^{n+1}\right)$ and $\widetilde{V}_{4}\left(\mathbb{R}^{n+1}\right)$, the one on the former manifold not being compatible with its known hypercomplex structure.
\end{abstract}

\section{INTRODUCTION}

The main motivation for the present work is to understand some aspects of the Riemannian geometry of the focal set $F o c_{\mathbb{H} P}{ }^{n} \mathbb{C} P^{n}$, i.e. of the set of points in the quaternionic projective space $\mathbb{H} P^{n}$ that are critical values of the normal exponential map with respect to the totally geodesic submanifold $\mathbb{C} P^{n} \subset \mathbb{H} P^{n}$. Our starting point is the Habilitationsschrift of J. Berndt [7], [8], where in particular is proved that $F o c_{\mathbb{H} P} \mathbb{C} P^{n}$ fibers in circles over $G r_{2}\left(\mathbb{C}^{n+1}\right)$, the Grassmannian of 2 -planes in $\mathbb{C}^{n+1}$. In the simplest cases $n=1,2$, these focal points distribute on spheres $S^{1}, S^{5}$, respectively and $n=3$ thus seems to be the first significant case. Indeed, since $F o c_{\mathbb{H} P 3} \mathbb{C} P^{3}$ is a circle bundle over the Klein quadric $G r_{2}\left(\mathbb{C}^{4}\right) \cong \mathbb{C} Q^{4} \subset \mathbb{C} P^{5}$, both the classical projective geometry of $\mathbb{C} Q^{4}$ (described for example in [39], pp. 26-35) and its two quaternion Kähler structures [27] can be related to the total space $F_{O c_{\mathbb{H} P} P^{3}} \mathbb{C} P^{3}$. More generally, the twofold Kähler-Einstein and quaternion Kähler properties of the Grassmannian $G r_{2}\left(\mathbb{C}^{n+1}\right)$ suggested to us a link between the focal set $F o c_{\mathbb{H} P^{n}} \mathbb{C} P^{n}$ and the Sasakian-Einstein geometry of the induced Hopf bundles considered in our previous work [31.

1991 Mathematics Subject Classification. 53C15, 53C25, 53C55.

Key words and phrases. Quaternion Kähler manifold, Sasakian structure, 3Sasakian structure, complex structure, focal set, Riemannian submersion, moment map, induced Hopf bundle. 
This paper begins by giving an explicit identification of the focal set $F o c_{\mathbb{H} P^{n}} \mathbb{C} P^{n}$ with the zero level set of the moment map $\mu$ associated to the diagonal $U(1)$ - action on $\mathbb{H} P^{n}$. This is the simplest isometric action giving examples of reductions in quaternion Kähler geometry, and it is well-known that the reduced manifold is precisely the quaternion Kähler Wolf space $G r_{2}\left(\mathbb{C}^{n+1}\right)[20]$, [21]. According to the notation in use, we write $\mathbb{H} P^{n} / / / U(1)=G r_{2}\left(\mathbb{C}^{n+1}\right)$ to indicate this reduction procedure; this has been proved to be the unique one with respect to isometric circle actions and positive quaternion Kähler manifolds [3], [4]. Next, the diagonal $S p(1)$-action on $\mathbb{H} P^{n}$ has as reduction the quaternion Kähler manifold $\mathbb{H} P^{n} / / S p(1)=\widetilde{G} r_{4}\left(\mathbb{R}^{n+1}\right)$, the Grassmannian of oriented 4-planes in $\mathbb{R}^{n+1}$. The zero set of the corresponding moment map $\nu: \mathbb{H} P^{n} \rightarrow \mathbb{H}$ can also be related to focal sets in $\mathbb{H} P^{n}$. (For these identifications cf. Theorems 3.1, 3.2).

On the other hand, the zero level sets $\mu^{-1}(0)$ and $\nu^{-1}(0)$ of these moment maps $\mu$ and $\nu$ can be identified with the total spaces of some induced Hopf $S^{1}$-bundles. This enables us to define on them a SasakianEinstein and a 3-Sasakian metric, respectively, and hence to find a diagram where these zero sets $\mu^{-1}(0)$ and $\nu^{-1}(0)$ are base spaces of $S^{3}$ bundles, now induced by the Hopf fibration $S^{4 n+3} \rightarrow \mathbb{H} P^{n}$. The corresponding total spaces are the Stiefel manifolds $V_{2}\left(\mathbb{C}^{n+1}\right)$ and $\widetilde{V}_{4}\left(\mathbb{R}^{n+1}\right)$, respectively. The 3-Sasakian structure of the fibers $S^{3}$ and the Sasakian structure of the base spaces $\mu^{-1}(0)$ and $\nu^{-1}(0)$ allow to construct a complex structure on $V_{2}\left(\mathbb{C}^{n+1}\right)$ and on $\widetilde{V}_{4}\left(\mathbb{R}^{n+1}\right)$, whose definition is very much in the Calabi-Eckmann spirit. On the former Stiefel manifold, this complex structure is not compatible with its standard hypercomplex structure, obtained in [2], [14], [24]. On the other hand, both $V_{2}\left(\mathbb{C}^{n+1}\right)$ and $\widetilde{V}_{4}\left(\mathbb{R}^{n+1}\right)$ are total spaces of framed bundles in Hopf surfaces over Kähler Einstein manifolds. Then these complex structures can be seen to belong to one-parameter families, also suitable for some exceptional cases, as shown in a forthcoming paper, [32].

Acknowledgements. This paper was written while the first named author was visiting the University La Sapienza of Rome and the Max Planck Institut für Mathematik in Bonn. He thanks both institutions for financial support and for hospitality. The second author acknowledges support from MURST project "Proprietà geometriche delle varietà reali e complesse".

Both authors thank Victor Vuletescu for some helpful discussions, and John C. Wood who accepted to correct our English and patiently read the whole paper. 


\section{Preliminaries}

We collect here some definitions and basic facts that will be used throughout the paper.

Let $(M, g)$ be a Riemannian manifold and let $N$ be an isometrically immersed submanifold. The critical values of the restriction $\exp _{T^{\perp} N}$ of the exponential map of $M$ to the normal bundle of $N$ are called focal points of $N$ and the set of all focal points, here denoted by $F_{o c} M$, is called the focal set of $N$ (see for example [18] p. 23, [19], p. 227, 228] p. 283). The focal set of a submanifold may not be a submanifold: for smooth plane curves and for regular surfaces in $\mathbb{R}^{3}$ the focal sets are respectively the evolutes and the surfaces of the centres, and both of them can have singular points, cf. [19], pp. 237-238 and p. 232. However, for the examples considered in the present paper all the focal sets turn out to be smooth. Indeed, it seems that not many focal sets of Riemannian submanifolds have been explicitly determined, but we can mention totally geodesic spheres in spheres [33], and hypersurfaces in space forms [16, [17.

We are mainly interested in $F o c_{\mathbb{H} P^{n}} \mathbb{C} P^{n}$, the focal set of the totally geodesic $\mathbb{C} P^{n}$ in the quaternionic projective space $\mathbb{H} P^{n}$. This focal set appears in J. Berndt's work [7], [8], denoted there by $Q^{n}$, and studied in connection with both the complex Kähler and the quaternion Kähler structure of $G r_{2}\left(\mathbb{C}^{n+1}\right)$, the Grassmannian of complex two-planes in $\mathbb{C}^{n+1}$.

Two geometric structures which appear naturally in our context are the Sasakian and the 3-Sasakian ones. We briefly recall the definitions, referring the reader to the survey [11] for further information.

A Sasakian manifold is a $(2 n+1)$-dimensional Riemannian manifold $(S, g)$ equipped with a unitary Killing vector field $\xi$ such that the field of endomorphisms $\varphi:=\nabla \xi$ satisfies the differential equation

$$
\nabla \varphi=I d \otimes \eta-g \otimes \xi
$$

where $\nabla$ is the Levi-Civita connection of $g$ and $\eta$ is the dual 1 -form of $\xi$. The data of a Sasakian structure on the manifold $S$ is easily seen to be equivalent to the requirement that the cone metric $d r^{2}+r^{2} g$, on $\mathbb{R}_{+} \times S$ have holonomy contained in $U(n+1)$. Note that $\eta$ is a contact form on $S$, hence $\xi$ is its Reeb field. In the simplest example, the Euclidean sphere $S^{2 n+1}$, the Killing vector field is $\xi=-J U, J$ being the standard complex structure of $\mathbb{C}^{n+1}$ and $U$ the unit outward normal to the sphere.

More generally, we look at the induced Hopf $S^{1}$-bundle $\pi: V \rightarrow$ $M$, over a smooth submanifold of $\mathbb{C} P^{N}$. Its total space $V$ carries a Sasakian structure $(V, g, \xi)$ induced from the one of $\left(S^{2 N+1}\right.$, can $)$. If 
the Fubini-Study metric of $\mathbb{C} P^{N}$ induces an Einstein metric $h$ with Einstein constant $\alpha$ on $M$, it can be seen (cf. [31], lemma 1) that the Ricci tensor of the metric $g$ has the form

$$
\operatorname{Ric}(g)=\lambda g+\mu \eta \otimes \eta,
$$

with $\lambda=\alpha-2, \mu=\operatorname{dim} V+1-\alpha$. This is known as the $\eta$-Einstein property in Sasakian geometry and, following S. Tanno [38], an $\eta$-Einstein Sasakian metric can be deformed to a Sasakian-Einstein one by setting

$$
g^{\prime}=A g+A(A-1) \eta \otimes \eta
$$

so that $\xi^{\prime}=A^{-1} \xi$ with $A=\frac{\lambda+2}{\operatorname{dim} V+1}$. Now $\pi$ is a Riemannian submersion with respect to the metrics $h$ and $g$; thus to have a Riemannian submersion with respect to $g^{\prime}$, we must consider the scaled metric $h^{\prime}=A h$ on the base $M$.

As Sasakian geometry may be viewed as the odd-dimensional counterpart of Kähler geometry, the odd-dimensional counterparts of hyperKähler manifolds are 3-Sasakian ones (cf. [11]). More precisely, a $(4 n+3)$-dimensional Riemannian manifold $(\bar{S}, g)$ is said to be 3 Sasakian if it is endowed with three mutually orthogonal unit Killing vector fields $\xi_{1}, \xi_{2}, \xi_{3}$, each one defining a Sasakian structure and satisfying the conditions:

$$
\left[\xi_{1}, \xi_{2}\right]=2 \xi_{3}, \quad\left[\xi_{2}, \xi_{3}\right]=2 \xi_{1}, \quad\left[\xi_{3}, \xi_{1}\right]=2 \xi_{2}
$$

As above, an equivalent definition requires that the cone metric $d r^{2}+$ $r^{2} g$ on $\mathbb{R}_{+} \times S$ be hyperkähler, i.e. its holonomy group be contained in $S p(n+1)$. 3-Sasakian manifolds are necessarily Einstein with positive scalar curvature and their Einstein constant is $4 n+2$. Given a positive quaternion Kähler manifold $P$, one constructs its Kähler-Einstein twistor space $Z_{P}$ and then an $S^{1}$ bundle over it whose Chern class is, up to torsion, that of an induced Hopf bundle. The total space $S$ thus obtained is an $S O(3)$-principal bundle over $P$ with 3-Sasakian structure. Moreover, all three fibrations involved are Riemannian submersions.

We recall now two basic moment maps of quaternion Kähler geometry. Let $\left[h_{0}: h_{1}: \ldots: h_{n}\right]$ be the homogeneous coordinates on $\mathbb{H} P^{n}$ : for each $a=0,1, \ldots, n$, we shall write the complex and real components of $h_{a}$ as follows:

$$
h_{a}=z_{a}+w_{a} j=\alpha_{a}+\beta_{a} i+\gamma_{a} j+\delta_{a} k
$$

where $z_{a}=\alpha_{a}+\beta_{a} i$, and $w_{a}=\gamma_{a}+\delta_{a} i$. The first moment map, induced by the diagonal action of $U(1)$ on $\mathbb{H} P^{n}$ is at the hyperkähler level of 
the bundle $\mathbb{H}^{n+1} \backslash\{0\} \rightarrow \mathbb{H} P^{n}$, given by:

$$
\mu: \mathbb{H}^{n+1} \backslash\{0\} \rightarrow \mathbb{H}, \quad \mu=\sum_{a=0}^{n} \bar{h}_{a} i h_{a}
$$

On $\mathbb{H} P^{n}$, one has to regard the corresponding moment map $\mu_{q K}$ as a section of $S^{2} H$, the rank 3 vector bundle of the compatible almost complex structures. In fact, by using the metric, $\mu_{q K}$ appears as a 2 -form solution of

$$
d \mu_{q K}=i_{X} \Omega
$$

where $\Omega$ is the Kähler 4 -form and $X$ the Killing vector field generating the $U(1)$-action: [20], [1]. However, since the zero level sets $\mu^{-1}(0) \subset \mathbb{H}^{n+1}$ and $\mu_{q K}^{-1}(0) \subset \mathbb{H} P^{n}$ correspond to each other in the bundle projection $\mathbb{H}^{n+1} \backslash\{0\} \rightarrow \mathbb{H} P^{n}$, we shall refer to the definition $\mu=\sum_{a=0}^{n} \bar{h}_{a} i h_{a}$, whose zero set makes sense also when the $h_{a}$ are the homogeneous coordinates of $\mathbb{H} P^{n}$ [11. The reduced manifold $\mu_{q K}^{-1}(0) / U(1)$ turns out to be the quaternion Kähler Wolf space $\frac{S U(n+1)}{S(U(n-1) \times U(2))} \cong G r_{2}\left(\mathbb{C}^{n+1}\right)$ [20, 21].

The second moment map to be considered, generated by the action of $S p(1)$, is (again at the hyperkähler level):

$$
\nu: \mathbb{H}^{n+1} \backslash\{0\} \rightarrow \mathbb{H}^{3}, \quad \nu=\left(\sum_{a=0}^{n} \bar{h}_{a} i h_{a}, \sum_{a=0}^{n} \bar{h}_{a} j h_{a}, \sum_{a=0}^{n} \bar{h}_{a} k h_{a}\right),
$$

and its corresponding quaternion Kähler moment map $\nu_{q K}$ can be viewed as a triple of 2 -forms associated to the frame of Killing vector fields defining the $S p(1)$-action. The corresponding reduced manifold $\nu_{q K}^{-1}(0) / S p(1)$ is now the Wolf space $\frac{S O(n+1)}{S O(n-3) \times S O(4)} \cong \widetilde{G r} r_{4}\left(\mathbb{R}^{n+1}\right)$, the Grassmannian of oriented four-planes of $\mathbb{R}^{n+1}$.

\section{Statement of Results}

Theorem 3.1. (i) The focal set $F$ oc $_{\mathbb{H} P^{n}} \mathbb{C} P^{n}$ coincides with $\mu^{-1}(0)$, and it is isometric to the total space of the induced Hopf $S^{1}$-bundle via the Plücker embedding $G r_{2}\left(\mathbb{C}^{n+1}\right) \hookrightarrow \mathbb{C} P^{N}$.

(ii) The metric $g_{1}$, induced on $\mu^{-1}(0)$ by the Plücker embedding allows us to define on $\mu^{-1}(0)$ a Sasakian Einstein metric $g$.

(iii) The Stiefel manifold $V_{2}\left(\mathbb{C}^{n+1}\right)$ of orthonormal 2-frames in $\mathbb{C}^{n+1}$, diffeomorphic to the total space of the induced Hopf $S^{3}$-bundle via the embedding $\mu^{-1}(0) \subset \mathbb{H} P^{n}$, admits an (integrable) complex structure $J$, not compatible with the standard hypercomplex structure of $V_{2}\left(\mathbb{C}^{n+1}\right)$. 
We have a similar statement regarding the moment map $\nu$ induced by the action of $S p(1)$ on $\mathbb{H} P^{n}$. We need to consider the following mutually congruent, totally geodesic embeddings of $\mathbb{C} P^{n}$ in $\mathbb{H} P^{n}$.

$$
\begin{aligned}
& \mathbb{C} P_{i}^{n}=\left\{h \in \mathbb{H} P^{n} ; \gamma_{a}=\delta_{a}=0, a=1, \ldots, n\right\}, \\
& \mathbb{C} P_{j}^{n}=\left\{h \in \mathbb{H} P^{n} ; \beta_{a}=\delta_{a}=0, a=1, \ldots, n\right\}, \\
& \mathbb{C} P_{k}^{n}=\left\{h \in \mathbb{H} P^{n} ; \beta_{a}=\gamma_{a}=0, a=1, \ldots, n\right\}:
\end{aligned}
$$

here $\mathbb{C} P_{i}^{n}$ is the standard $\mathbb{C} P^{n}$ appearing in Theorem 3.1.

Theorem 3.2. (i) The zero level set $\nu^{-1}(0)$ coincides with the intersection $M=F o c_{\mathbb{H} P^{n}} \mathbb{C} P_{i}^{n} \cap F o c_{\mathbb{H} P^{n}} \mathbb{C} P_{j}^{n} \cap F o c_{\mathbb{H} P^{n}} \mathbb{C} P_{k}^{n}$ and is isometric to the total space of the induced Hopf $S^{1}$-bundle over the Fano manifold $Z_{\widetilde{G r}_{4}\left(\mathbb{R}^{n+1}\right)}$, via the embeddings $Z_{\widetilde{G r}_{4}\left(\mathbb{R}^{n+1}\right)} \hookrightarrow G r_{2}\left(\mathbb{C}^{n+1}\right) \hookrightarrow \mathbb{C} P^{N}$, the first of which is defined by regarding $Z_{\widetilde{G r}_{4}\left(\mathbb{R}^{n+1}\right)}$ as the space of totally isotropic two-planes in $\mathbb{C}^{n+1}$. This isometry allows the construction of a Sasakian Einstein metric on $\nu^{-1}(0)$, and identifies it with the homogeneous 3-Sasakian manifold $S O(n+1) /(S O(n-3) \times S p(1))$.

(ii) The Stiefel manifold $\widetilde{V}_{4}\left(\mathbb{R}^{n+1}\right)$ admits an (integrable) complex structure, projecting to the complex structure of $Z_{\widetilde{G r}_{4}\left(\mathbb{R}^{n+1}\right)}$.

Here $Z_{\widetilde{G r}_{4}\left(\mathbb{R}^{n+1}\right)}$ is the twistor space of the quaternion Kähler Wolf space $\widetilde{G r} r_{4}\left(\mathbb{R}^{n+1}\right)$ given by the $S p(1)$ reduction on $\mathbb{H} P^{n} \cdot Z_{\widetilde{G r}}\left(\mathbb{R}^{n+1}\right)$ is known to be a complex submanifold of the Kähler-Einstein Grassmannian $G r_{2}\left(\mathbb{C}^{n+1}\right)$, see for example [25] p. 14 or [23] p. 702 .

Statements 3.1, 3.2 give, in particular, some fibrations that can be collected into a diagram as follows. Here $V_{k}\left(\mathbb{C}^{n+1}\right)$ and $\widetilde{V}_{k}\left(\mathbb{R}^{n+1}\right)$ denote the Stiefel manifolds of $k$-frames in $\mathbb{C}^{n+1}$ and of oriented $k$-frames in $\mathbb{R}^{n+1}$, respectively.

Corollary 3.1. There is a commutative diagram

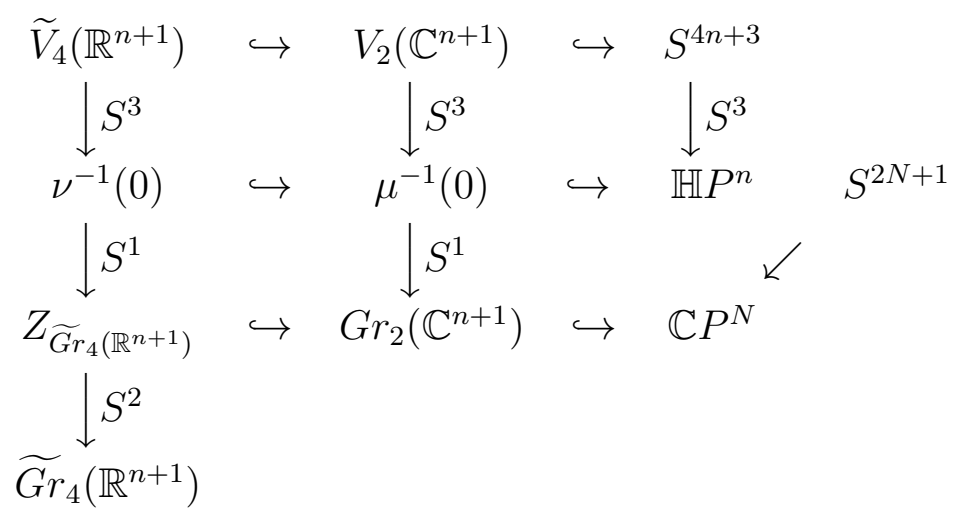


of principal $S^{1}$ and $S^{3}$-bundles and Riemannian submersions. The zero level sets $\mu^{-1}(0)$ and $\nu^{-1}(0)$ are thus total spaces of induced Hopf $S^{1}$ bundles and, as such, are minimal submanifolds of the sphere $S^{2 N+1}$.

\section{THE PROOFS}

Proof of Theorem 3.1 (i) and (ii). The first observation is:

$F o c_{\mathbb{H} P} \mathbb{C} P^{1}$ is the level set of the moment map associated to the standard $U(1)$-action on $\mathbb{H} P^{1}$.

In the homogeneous coordinates $\left[h_{0}: h_{1}\right]$ of $\mathbb{H} P^{1}$, using the notations from formula (2.1), $\mathbb{C} P^{1}$ is given by:

$$
w_{0}=0, w_{1}=0 \text { or } \gamma_{0}=\delta_{0}=\gamma_{1}=\delta_{1}=0 .
$$

The pair $\mathbb{C} P^{1} \subset \mathbb{H} P^{1}$ can be identified with $S^{2} \subset S^{4}$, and the focal set of a totally geodesic sphere $S^{p}$ in $S^{n}$ is the unit $S^{n-p-1}$ in the $(n-p)$-dimensional orthogonal complement of the $\mathbb{R}^{p+1}$ containing $S^{p}$ ([33], p. 286). This identification can be made explicit by using the coordinate $h=\alpha+\beta i+\gamma j+\delta k=h_{0} h_{1}^{-1}$ in the affine line $h_{1} \neq 0$, whose corresponding real coordinates are:

$$
\begin{aligned}
& \alpha=\frac{\alpha_{0} \alpha_{1}+\beta_{0} \beta_{1}+\gamma_{0} \gamma_{1}+\delta_{0} \delta_{1}}{r^{2}}, \beta=\frac{\alpha_{1} \beta_{0}-\alpha_{0} \beta_{1}-\gamma_{0} \delta_{1}+\gamma_{1} \delta_{0}}{r^{2}}, \\
& \gamma=\frac{\alpha_{1} \gamma_{0}-\alpha_{0} \gamma_{1}-\delta_{0} \beta_{1}+\beta_{0} \delta_{1}}{r^{2}}, \delta=\frac{\alpha_{1} \delta_{0}-\alpha_{0} \delta_{1}-\gamma_{1} \beta_{0}+\gamma_{0} \delta_{1}}{r^{2}},
\end{aligned}
$$

where $r^{2}=\alpha_{1}^{2}+\beta_{1}^{2}+\gamma_{1}^{2}+\delta_{1}^{2}$. If $\left(x_{1}, \ldots, x_{5}\right)$ are the standard coordinates on $\mathbb{R}^{5}$, the (inverse) stereographic projection $\mathbb{R}^{4} \rightarrow S^{4}$ reads:

$$
(\alpha, \beta, \gamma, \delta) \mapsto\left(x_{1}, \ldots, x_{5}\right)=\left(\frac{2 \alpha}{r^{2}+1}, \frac{2 \beta}{r^{2}+1}, \frac{2 \gamma}{r^{2}+1}, \frac{2 \delta}{r^{2}+1}, \frac{r^{2}-1}{r^{2}+1}\right) .
$$

Thus, if $x_{3}=x_{4}=0$ are the equations of a totally geodesic $S^{2}$ in $S^{4}$, the focal set of $S^{2}$ in $S^{4}$ is the $S^{1}$ given by the equations $x_{1}=x_{2}=x_{5}=0$, corresponding to

$$
\alpha=\beta=0, \gamma^{2}+\delta^{2}=1 .
$$

On the other hand, the moment map $\mu$ on $\mathbb{H} P^{1}$ can be written in the non-homogeneous coordinate $h=h_{0} h_{1}^{-1}$ as $\mu=\bar{h} i h+i$. Its level set $\mu^{-1}(0)$ is thus:

$$
\alpha_{1}^{2}+\beta_{1}^{2}-\gamma_{1}^{2}-\delta_{1}^{2}+1=0, \alpha \delta-\beta \gamma=0, \alpha \gamma+\beta \gamma=0,
$$


and the systems (4.3) and (4.4) are equivalent.

We now treat the case $n>1$. From the definition of the focal set, we only need to look at geodesics normal to $\mathbb{C} P^{n}$. These are normal to all the complex projective lines $\mathbb{C} L^{1} \subset \mathbb{C} P^{n}$, thus their focal points with respect to $\mathbb{C} P^{n}$ are also focal points for all the lines $\mathbb{C} L^{1}$ contained in $\mathbb{C} P^{n}$. Any such line $\mathbb{C} L^{1}$ in $\mathbb{H} P^{n}$ belongs to a unique quaternionic projective line $\mathbb{H} L^{1}$ and the latter is totally geodesic in $\mathbb{H} P^{n}$. It follows that any geodesic that is normal to a $\mathbb{C} L^{1}$ and tangent to the corresponding $\mathbb{H} L^{1}$ at a given point, remains tangent to $\mathbb{H} L^{1}$ for its entire length. Hence, if we show that through any point $x$ of $\mathbb{C} P^{n}$ and for any $v \in T_{x}^{\perp} \mathbb{C} P^{n}$ there exists a projective quaternionic line $\mathbb{H} L^{1}$ containing $v$, we may deduce:

$F_{o c_{\mathbb{H} P^{n}}} \mathbb{C} P^{n}$ is the union of all the focal sets $F o c_{\mathbb{H} L^{1}} \mathbb{C} L^{1}$.

This can be seen from the diagram

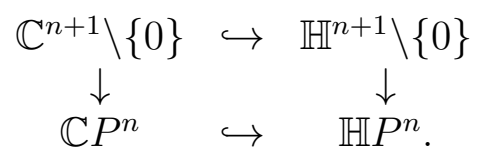

by looking at the complex planes $L_{2}^{\mathbb{C}}$ in $\mathbb{C}^{n+1}$ containing the fibre $\mathbb{C}^{*}$ and the corresponding hypercomplex 2-planes $L_{2}^{\mathbb{H}}$ in $\mathbb{H}^{n+1}$ containing the fibre $\mathbb{H}^{*}$. Observe that, for any vector $\vec{v} \in \mathbb{H}^{n+1}$ which is normal to the standard embedded $\mathbb{C}^{n+1}$, there exist a $L_{2}^{\mathbb{C}}$ and a $L_{2}^{\mathbb{H}}$ with $\operatorname{span}_{\mathbb{R}}\left\{L_{2}^{\mathbb{C}}, \vec{v}\right\} \subset L_{2}^{\mathbb{H}}$ : indeed, if $\mathbb{H}^{n+1}=\operatorname{span}_{\mathbb{H}}\left\{\overrightarrow{e_{0}}, \ldots, \overrightarrow{e_{n}}\right\}$ and $\mathbb{C}^{n+1}=\operatorname{span}_{\mathbb{C}}\left\{\overrightarrow{e_{0}}, \ldots, \overrightarrow{e_{n}}\right\}$, we have:

$$
\vec{v} \perp \mathbb{C}^{n+1} \text { if and only if } \vec{v}=\sum_{a=0}^{n} \lambda_{a} j \overrightarrow{e_{a}}+\mu_{a} k \overrightarrow{e_{a}} \text {. }
$$

Hence, if

$$
\vec{w}=-j \vec{v}=\sum_{a=0}^{n} \lambda_{a} \overrightarrow{e_{a}}-\mu_{a} i \overrightarrow{e_{a}}
$$

we obtain

$$
L_{2}^{\mathbb{C}}=\operatorname{span}_{\mathbb{C}}\left\{\overrightarrow{e_{0}}, \vec{w}\right\}
$$

and

$$
L_{2}^{\mathbb{H}}=\operatorname{span}_{\mathbb{R}}\left\{\overrightarrow{e_{0}}, i \overrightarrow{e_{0}}, j \overrightarrow{e_{0}}, k \overrightarrow{e_{0}}, \vec{w}, i \vec{w}, j \vec{w}, k \vec{w}\right\}
$$

satisfy the condition.

The identification of $F o c_{\mathbb{H} P^{n}} \mathbb{C} P^{n}$ with $\mu^{-1}(0)$ is then completed by the following observation: 
The subset $\mu^{-1}(0) \subset \mathbb{H} P^{n}$ is the union of the zero sets of the moment maps $\mu_{1}$ associated to the standard $U(1)$-action on all the projective quaternionic lines $\mathbb{H} L^{1} \subset \mathbb{H} P^{n}$.

Let $\left\{p_{0}, p_{1}, \ldots, p_{n}\right\}$ be the canonical frame of $\mathbb{H} P^{n}$ with unit point $u$ and let $\left[h_{0}: \ldots: h_{n}\right]$ be the homogeneous coordinates with respect to this frame. Accordingly, the moment map reads $\mu(h)=\sum_{a=0}^{n} \bar{h}_{a} i h_{a}$. Fix a $q \in \mu^{-1}(0)$, i.e. $\sum_{a=0}^{n} \bar{q}_{a} i q_{a}=0$, and note that $q$ cannot be real; however, we may suppose $q_{0} \neq 0$.

Let $\mathbb{H} L^{1} \cong \mathbb{H} P^{1}$ be the quaternionic projective line through $q$ and $p_{1}$. To compare the intersection $\mu^{-1}(0) \cap \mathbb{H} L^{1}$ with the zero level set of the moment map in $\mathbb{H} L^{1} \cong \mathbb{H} P^{1}$, we change the frame in $\mathbb{H} P^{n}$ as follows. We want new homogeneous coordinates $\left[k_{0}: k_{1}: \ldots: k_{n}\right]$ such that $q=[1: j: 0: \ldots: 0]$ and the coordinates of $p_{1}, \ldots, p_{n}$ remain unchanged. With the new unit point $v=\left[q_{0}: 1:-1:-1: \ldots:-1\right]$, the matrix of the change of coordinates is:

$$
A=\left(\begin{array}{ccccc}
q_{0} & 0 & 0 & \ldots & 0 \\
q_{1}-j & 1 & 0 & \ldots & 0 \\
q_{2} & 0 & -1 & \ldots & 0 \\
\ldots \ldots & \ldots & \ldots & \ldots & \ldots \\
q_{n} & 0 & 0 & \ldots & -1
\end{array}\right)
$$

Thus, ${ }^{t}\left[h_{0}: \ldots: h_{n}\right]=A^{t}\left[k_{0}: \ldots: k_{n}\right]$, and the moment map is:

$$
\mu=\sum_{a=0}^{n} \overline{h_{a}} i h_{a}=\overline{k_{0}}\left(\sum_{a=0}^{n} \overline{q_{a}} i q_{a}\right) k_{0}-\overline{k_{0}} j i j k_{0}-\sum_{b=1}^{n} \overline{k_{b}} i k_{b}=-\sum_{b=0}^{n} \overline{k_{b}} i k_{b} .
$$

It follows that $\mu^{-1}(0)$ is invariant under these projective changes of coordinates. As the coordinates on $\mathbb{H} L^{1}$ are $\left[k_{0}: k_{1}\right]$, its moment map is $\mu_{1}=-\overline{k_{0}} i k_{0}-\overline{k_{1}} i k_{1}$ with the same level set $\mu_{1}^{-1}(0)$ described for $\mathbb{H} P^{1}$. This establishes the inclusion $\left.\mu^{-1}(0)\right|_{\mathbb{H} L^{1}} \subset \mu_{1}^{-1}(0)$. As the converse inclusion is clear, for any projective line $\mathbb{H} L^{1}$ in $\mathbb{H} P^{n}$, the proof of the identification $F o c_{\mathbb{H} P^{n}} \mathbb{C} P^{n}=\mu^{-1}(0)$ is complete.

Next, we prove that $F_{o c_{\mathbb{H}} P^{n}} \mathbb{C} P^{n}$ is isometric to the total space of the induced Hopf bundle over the Grassmannian $G r_{2}\left(\mathbb{C}^{n+1}\right)$. In fact, since $F o c_{\mathbb{H} P^{n}} \mathbb{C} P^{n}$ is simply connected $([7]$, p. 17), the existence of a diffeomorphism with the induced Hopf bundle is a consequence of the following observation:

Let $\pi: P \rightarrow G r_{2}\left(\mathbb{C}^{n+1}\right)$ be a principal circle bundle with simply connected $P$. Then $P$ is diffeomorphic to the total space of the induced 
Hopf bundle of $S^{2 N+1} \rightarrow \mathbb{C} P^{N}, N=\left(\begin{array}{c}n+1 \\ 2\end{array}\right)-1$, via the Plücker embedding $G r_{2}\left(\mathbb{C}^{n+1}\right) \hookrightarrow \mathbb{C} P^{N}$.

In fact, the principal $S^{1}$-bundles over the base $B$ are classified by their Chern class in $H^{2}(B, \mathbb{Z})$. Since $H^{2}\left(G r_{2}\left(\mathbb{C}^{n+1}\right), \mathbb{Z}\right) \cong \mathbb{Z}$ is generated by the class of the Kähler form, one can denote by $P_{a}$ the $S^{1}$-bundle over $G r_{2}\left(\mathbb{C}^{n+1}\right)$ associated to $a \in \mathbb{Z}$. Observe that $P_{1}$ (resp. $P_{-1}$ ) is the circle bundle associated to the canonical line bundle $O_{G r_{2}\left(\mathbb{C}^{n+1}\right)}(1)$ (resp. its dual $O_{G r_{2}\left(\mathbb{C}^{n+1}\right)}(-1)$ ). But dual complex line bundles are diffeomorphic as real vector bundles. Hence $P_{1}$ is diffeomorphic to $P_{-1}$.

Let us now show that if $P_{a}$ is simply connected, then $a= \pm 1$. From $\pi_{1}(P)=0$, we have $H^{1}(P, \mathbb{Z})=0$ and $H^{2}(P, \mathbb{Z})$ torsion free. Thus the Gysin sequence of the $S^{1}$-bundle $\pi$ :

$$
\begin{aligned}
0 \rightarrow H^{1}(P, \mathbb{Z}) & \rightarrow H^{0}\left(G r_{2}\left(\mathbb{C}^{n+1}\right), \mathbb{Z}\right) \rightarrow H^{2}\left(G r_{2}\left(\mathbb{C}^{n+1}\right), \mathbb{Z}\right) \\
& \rightarrow H^{2}(P, \mathbb{Z}) \rightarrow 0
\end{aligned}
$$

reduces to:

$$
0 \longrightarrow \mathbb{Z} \stackrel{\cup c_{1}}{\longrightarrow} \mathbb{Z} \longrightarrow H^{2}(P, \mathbb{Z}) \longrightarrow 0
$$

where $c_{1}$ is the Chern class of $\pi$. Hence, $c_{1} \neq \pm 1$ implies $H^{2}(P, \mathbb{Z}) \cong \mathbb{Z}_{n}$ for some $n \geq 2$. Consequently, $c_{1}$ is, up to sign, the Chern class of the induced Hopf bundle.

We now look at the metric $g_{1}$ inherited from $\left(S^{2 N+1}\right.$, can $)$ by the total space $F_{0 c_{\mathbb{H} P^{n}}}\left(\mathbb{C} P^{n}\right)$ of the induced Hopf bundle and at the metric $g$ induced on $F o c_{\mathbb{H} P^{n}}\left(\mathbb{C} P^{n}\right)$ from $\mathbb{H} P^{n}$. Note that both $\left(F o c_{\mathbb{H} P^{n}}\left(\mathbb{C} P^{n}\right), g_{1}\right)$ and $\left(F o c_{\mathbb{H} P^{n}}\left(\mathbb{C} P^{n}\right), g\right)$ are Riemannian submersions with geodesic fibres $S^{1}$ over $G r_{2}\left(\mathbb{C}^{n+1}\right)$ (cf. [31] for $g_{1}$ and [7] for $g$ ). Thus Theorem 9.59 in [6], p. 249, can be used to conclude that $g=g_{1}$. This completes the proof of (i) and (ii) in Theorem 3.1.

Remark 4.1. An alternative way of recognizing that $F o c_{\mathbb{H} P^{n}} \mathbb{C} P^{n}=$ $\mu^{-1}(0)$ is to look at the standard isometric action of $S U(n+1)$ on $\mathbb{H} P^{n}$ and to see that the two subsets are obtained as homogeneous spaces of $S U(n+1)$ with the same isotropy groups. The homogeneous space is in fact $\frac{S U(n+1)}{S U(2) \times S U(n-1)}$, which was shown in [7], p. 17, to be a singular orbit of the action of $S U(n+1)$ (it was denoted there by $Q^{n}$ ). A similar description of $\mu^{-1}(0)$ as a homogeneous space is given in [1], $\mathrm{p}$. 65 , in relation with the problem of studying local compatible complex structures in $\mathbb{H} P^{n}$. Compare also with [36], p. 171, where $\frac{S U(n+1)}{S U(2) \times S U(n-1)}$ is called the "Grassmannian of oriented two-planes" of $\mathbb{C}^{n+1}$. 
Remark 4.2. We proved in [31] that the metric $g_{1}$ is Sasakian and $\eta$ Einstein, and this is in accordance with formulas following Proposition 9 in [7]. As for the extrinsic geometry of $\operatorname{Foc}_{\mathbb{H} P^{n}}\left(\mathbb{C} P^{n}\right)$, J. Berndt proves (Corollary 1 in [7]) that the immersion in $\mathbb{H} P^{n}$ is minimal. It is interesting to observe that the same holds for the immersion $\left(F o c_{\mathbb{H} P^{n}} \mathbb{C} P^{n}, g_{1}\right)$ in $\left(S^{2 N+1}, c a n\right)$, see [31].

Proof of Theorem 3.2 (i). The identification of $\nu^{-1}(0)$ with the intersection $M$ is an immediate consequence of the definition of $\nu$ and of the first statement of Theorem 3.1. To see that $\nu^{-1}(0)$ is isometric to the induced Hopf bundle discussed above, note that the last observation in the proof of Theorem 3.1 (i) and (ii) still holds for principal circle bundles with simply connected $P$ over any complex algebraic projective submanifold $B$ of $\mathbb{C} P^{N}$ with $H^{2}(B, \mathbb{Z}) \cong \mathbb{Z}$. This applies in particular to $B=Z_{\widetilde{G r}_{4}\left(\mathbb{R}^{n+1}\right)}$, as soon as one recognizes that $\nu^{-1}(0)$ is simply connected. To see this, observe that $\nu^{-1}(0)$ can be regarded as the homogeneous space $\frac{S O(n+1)}{S O(n-3) \times S p(1)}$ via the transitive action of $S O(n+1)$ on $\nu^{-1}(0)$. This last action comes, in fact, from the natural action of $S O(n+1)$ on $\mathbb{H} P^{n}$ and J. Berndt's observation that $S U(n+1)$ acts transitively on $F_{o c_{\mathbb{H} P^{n}}}\left(\mathbb{C} P^{n}\right)$. His argument can be triplicated to produce transitive actions of groups $S U_{i}(n+1), S U_{j}(n+1), S U_{k}(n+1)$ (the first one is the standard $S U(n+1)$ in $S p(n+1)$, the other two are similarly defined by interchanging the rôles of the unit quaternions $i, j, k)$ on the zero level sets $\mu_{i}^{-1}(0), \mu_{j}^{-1}(0), \mu_{k}^{-1}(0)$. The isotropy subgroup of the action on $\nu^{-1}(0)$ at the point $[1: i: j: k: 0 \ldots: 0]$ is then $S p(1) \times S O(n-3)$. The homotopy sequence associated to the homogeneous manifold $\frac{S O(n+1)}{S p(1) \times S O(n-3)}$ thus shows that $\nu^{-1}(0)$ is simply connected, so that it is diffeomorphic with the total space of the induced Hopf bundle, now over the Fano manifold $Z_{\widetilde{G r}_{4}\left(\mathbb{R}^{n+1}\right)}$.

As an induced $S^{1}$-Hopf bundle, $\nu^{-1}(0)$ has a Sasakian $\eta$ - Einstein structure. This can be deformed to a Sasakian-Einstein metric, which is still a Riemannian submersion after rescaling the standard KählerEinstein metric of $Z_{\widetilde{G r} r_{4}\left(\mathbb{R}^{n+1}\right)}$ [31]. On the other hand, the composition of the fiberings

$$
\nu^{-1}(0) \stackrel{S^{1}}{\rightarrow} Z_{\widetilde{G r}_{4}\left(\mathbb{R}^{n+1}\right)} \stackrel{S^{2}}{\rightarrow} \widetilde{G r_{4}}\left(\mathbb{R}^{n+1}\right)
$$

is an $S O(3)$-bundle which endows $\nu^{-1}(0)$ with a 3-Sasakian structure via the inversion Theorem 4.6 of [10].

Proof of Theorem 3.1 (iii) and Theorem 3.2 (ii). In both cases we construct a complex structure on the total space of an $S^{3}$ bundle over a Sasakian manifold, induced by the Hopf bundle $S^{4 n+3} \rightarrow \mathbb{H} P^{n}$. 
Both Stiefel manifolds under discussion are induced Hopf $S^{3}$-bundles, as is easily recognized by regarding them as homogeneous spaces. Thus the induced homogeneous metrics make them Riemannian submersions with fibers $S^{3}$. More generally:

Proposition 4.1. Let $\pi: P \rightarrow B$ be a principal $S^{3}$-bundle induced by the Hopf bundle $S^{4 n+3} \rightarrow \mathbb{H} P^{n}$, and let $g^{B}, \nabla^{B}$ be the induced metric and Levi Civita connection on $B \subset \mathbb{H} P^{n}$. Assume that $B$ admits a Killing vector field $\xi$ such that $\varphi=\nabla^{B} \xi$ defines on $B$ a Sasakian structure Then $P$ admits an almost complex structure.

Proof. Let $g^{P}$ be the induced metric on $P \subset S^{4 n+3}$, so that $\pi$ is a Riemannian submersion. For any $X \in \mathcal{X}(B)$ we let $X^{*}$ be its horizontal lift on $P$. Let $\xi_{1}, \xi_{2}, \xi_{3}$ be the unit Killing vector fields which give the usual 3-Sasakian structure of the fibers $S^{3}$ and $\eta_{1}, \eta_{2}, \eta_{3}$ their duals with respect to the canonical metric of $S^{3}$. The $\xi_{i}$ may be viewed as vector fields on $P$. Let $\hat{\eta}_{i}$ be their dual forms with respect to the metric $g^{P}$. Restricted to any fibre, the $\hat{\eta}_{i}$ coincide with the $\eta_{i}$. The usual splitting $T P=\mathcal{V} \oplus \mathcal{H}$ into vertical and horizontal parts may be refined to:

$$
T P=\operatorname{span}\left\{\xi_{1}, \xi_{2}, \xi_{3}\right\} \oplus \operatorname{span}\left\{\xi^{*}\right\} \oplus \mathcal{H}^{\prime},
$$

where $\mathcal{H}^{\prime}$ represents the horizontal vector fields orthogonal to the horizontal lift $\xi^{*}$ of $\xi$..

Define the almost complex structure $J$ on $P$ by:

- $J \xi_{1}=\xi_{2}, \quad J \xi_{2}=-\xi_{1}$,

- $J \xi_{3}=\xi^{*}, J \xi^{*}=-\xi_{3}$,

- $J X^{*}=(\varphi X)^{*}$ for any $X \in \mathcal{X}(B)$ orthogonal to $\xi$.

Note that for $X \perp \xi, X^{*}$ is a section of $\mathcal{H}^{\prime}$. As the restriction of $\varphi$ to $\xi^{\perp}$ is an endomorphism of $\xi^{\perp}$, the last item in the definition is consistent. One easily shows that $J^{2}=-1$ and $J$ is compatible with $g$.

To discuss the integrability of the constructed $J$, we follow the discussion developed [15] for an almost hypercomplex structure, computing the Nijenhuis tensor field on all possible combinations of vertical and/or horizontal vector fields.

Note first that the horizontal distribution $\mathcal{H}$ is an $s p(1)$-connection in the induced Hopf $S^{3}$ bundle $P \rightarrow B$. This follows from the fact that the bracket of any horizontal $X^{*}$ with a vertical vector field is horizontal, a consequence of the Killing property of the $\xi_{i}$ with respect to $g^{P}$. Thus, in particular we get that for $i=1,2,3$ :

$$
\hat{\eta}_{k}\left[\xi_{i}, X^{*}\right]=\hat{\eta}_{k}\left[\xi_{i}, \xi^{*}\right]=0,
$$

for $k=1,2,3$. Now we can prove: 
Proposition 4.2. Assume that the curvature form $\Omega$ of the $s p(1)$ connection $\mathcal{H}$ satisfies the following conditions:

1) $\Omega\left((\varphi X)^{*},(\varphi Y)^{*}\right)=\Omega\left(X^{*}, Y^{*}\right)$, i.e. $\Omega$ is of type $(1,1)$ with respect to $J$,

2) $\Omega\left(X^{*}, \xi^{*}\right)=0$ for any $X \perp \xi$.

Then the almost complex structure $J$ is integrable.

Remark 4.3. Conditions 1), 2) in the former Proposition express a compatibility between the Sasakian structure of the base and the bundle structure of $P$. Since the vertical components of $\Omega$ are precisely the $d \hat{\eta}_{i}$, the two conditions give corresponding equations for $d \hat{\eta}_{i}$. Moreover, the condition $d \hat{\eta}_{i}\left((\varphi X)^{*},(\varphi Y)^{*}\right)=d \hat{\eta}_{i}\left(X^{*}, Y^{*}\right)$ is easily checked to be equivalent with $d \hat{\eta}_{i}\left((\varphi X)^{*}, Y^{*}\right)+d \hat{\eta}_{i}\left(X^{*},(\varphi Y)^{*}\right)=0$.

Now we can give the proof of the Proposition 4.2:

Proof. We compute the Nijenhuis tensor field of $J$ :

$$
[J, J]\left(A_{1}, A_{2}\right)=\left[A_{1}, A_{2}\right]+J\left[J A_{1}, A_{2}\right]+J\left[A_{1}, J A_{2}\right]-\left[J A_{1}, J A_{2}\right]
$$

for all possible pairs $\left(A_{1}, A_{2}\right)$, noting that, due to the tensorial character of $[J, J]$, when dealing with horizontal (resp. vertical) vector fields it is enough to work with basic ones (resp. with $\xi_{i}, i=1,2,3$ ).

1. Let first $A_{1}=X^{*}, A_{2}=Y^{*}, X, Y \perp \xi$. Denoting by $\hat{\eta}$ the dual of $\xi^{*}$ we get:

$$
\left[X^{*}, Y^{*}\right]=\left[X^{*}, Y^{*}\right]^{\prime}+\hat{\eta}\left(\left[X^{*}, Y^{*}\right]\right) \xi^{*}+\text { vertical part. }
$$

where the ' denotes the $\mathcal{H}^{\prime}$ part. By $\pi$-corelation, $\left[X^{*}, Y^{*}\right]=[X, Y]^{* \prime}$. Moreover, from $\hat{\eta}\left(X^{*}\right)=\hat{\eta}\left(Y^{*}\right)=0$ we get $\hat{\eta}\left(\left[X^{*}, Y^{*}\right]\right)=-d \hat{\eta}\left(X^{*}, Y^{*}\right)$. The vertical part of $\left[X^{*}, Y^{*}\right]$ must be of the form $\sum_{i=1}^{3} \hat{\eta}_{i}\left(\left[X^{*}, Y^{*}\right]\right) \xi_{i}$. Hence:

$$
\left[X^{*}, Y^{*}\right]=\left[X^{*}, Y^{*}\right]^{\prime}-d \hat{\eta}\left(X^{*}, Y^{*}\right) \xi^{*}-\sum_{i=1}^{3} d \hat{\eta}\left(X^{*}, Y^{*}\right) \xi_{i}
$$


By similar computations:

$$
\begin{aligned}
J\left[J X^{*}, Y^{*}\right] & =(\varphi[\varphi X, Y])^{* \prime}+d \hat{\eta}\left((\varphi X)^{*}, Y^{*}\right) \xi_{3}- \\
& -d \hat{\eta}_{1}\left((\varphi X)^{*}, Y^{*}\right) \xi_{2}+d \hat{\eta}_{2}\left((\varphi X)^{*}, Y^{*}\right) \xi_{1}-d \hat{\eta}_{3}\left((\varphi X)^{*}, Y^{*}\right) \xi^{*}, \\
J\left[X^{*}, J Y^{*}\right] & =(\varphi[X, \varphi Y])^{* \prime}+d \hat{\eta}\left(X^{*},(\varphi Y)^{*}\right) \xi_{3}- \\
& -d \hat{\eta}_{1}\left(X^{*},(\varphi Y)^{*}\right) \xi_{2}+d \hat{\eta}_{2}\left(X^{*},(\varphi Y)^{*}\right) \xi_{1}-d \hat{\eta}_{3}\left(X^{*},(\varphi Y)^{*}\right) \xi^{*}, \\
{\left[J X^{*}, J Y^{*}\right] } & =[\varphi X, \varphi Y]^{* \prime}-d \hat{\eta}\left((\varphi X)^{*},(\varphi Y)^{*}\right) \xi^{*} \\
& -\sum_{i=1}^{3} d \hat{\eta}_{i}\left((\varphi X)^{*},(\varphi Y)^{*}\right) \xi_{i} .
\end{aligned}
$$

Hence, using the $(1,1)$ character of $d \hat{\eta}_{i}$ and Remark 4.3, we find

$$
[J, J]\left(X^{*}, Y^{*}\right)=[\varphi X, \varphi Y]^{* \prime}-\left\{d \hat{\eta}\left(X^{*}, Y^{*}\right)-d \hat{\eta}\left((\varphi X)^{*},(\varphi Y)^{*}\right)\right\} \xi^{*} .
$$

As we know $[\varphi X, \varphi Y]+2 d \eta(X, Y) \xi=0$ (this is the normality condition of the Sasakian structure of $B$ ) the horizontal lift of this (null) tensor field is zero, hence also its component in $\mathcal{H}^{\prime}$ is zero. But this is precisely $[\varphi X, \varphi Y]^{* \prime}$.

On the other hand, on any Sasakian manifold one has:

$$
d \eta(X, Y)=g(X, \varphi Y), \quad \varphi^{2} X=-X+\eta(X) \xi,
$$

thus $d \eta(X, \varphi Y)+d \eta(\varphi X, Y)=0$ and $d \eta(\varphi X, \varphi Y)-d \eta(X, Y)=0$. By horizontally lifting these equations we obtain the annulation of the $\xi^{*}$ component, hence $[J, J]\left(X^{*}, Y^{*}\right)=0$.

2. Let now $A_{1}=X^{*}, A_{2}=\xi^{*}(X \perp \xi)$. Then:

$$
\begin{aligned}
{[J, J]\left(X^{*}, \xi^{*}\right) } & =\left[X^{*}, \xi^{*}\right]+J\left[J X^{*}, \xi^{*}\right]+J\left[X^{*}, J \xi^{*}\right]-\left[J X^{*}, J \xi^{*}\right]= \\
& =\left[X^{*}, \xi^{*}\right]+J\left[(\varphi X)^{*}, \xi^{*}\right]-J\left[X^{*}, \xi_{3}\right]+\left[(\varphi X)^{*}, \xi_{3}\right]= \\
& =\left[X^{*}, \xi^{*}\right]+J\left[(\varphi X)^{*}, \xi^{*}\right]
\end{aligned}
$$

as the last two brackets are zero by (4.5). As above, using Remark 4.3 and $d \hat{\eta}_{i}\left(X^{*}, \xi^{*}\right)=0$ (condition 2) in the statement) we obtain:

$$
[J, J]\left(X^{*}, \xi^{*}\right)=([X, \xi]+\varphi[\varphi X, \xi])^{* \prime}=0,
$$

because, as on a Sasakian manifold $\varphi \xi=0$, we can add in the paranthesis the terms $[X, \varphi \xi]-[\varphi X, \varphi \xi]$ obtaining $([X, \xi]+\varphi[\varphi X, \xi]+\varphi[X, \varphi \xi]-$ $[\varphi X, \varphi \xi])^{* \prime}=([\varphi, \varphi](X, \xi))^{* \prime}=0$ by the normality condition on $B$.

3. We now choose $A_{1}=X^{*}$ and $A_{2}=\xi_{i}(i=1,2)$. We have:

$$
[J, J]\left(X^{*}, \xi_{1}\right)=\left[X^{*}, \xi_{1}\right]+J\left[J X^{*}, \xi_{1}\right]+J\left[X^{*}, J \xi_{1}\right]-\left[J X^{*}, J \xi_{1}\right]=0,
$$


because $\mathcal{H}$ is a $\operatorname{sp}(1)$-connection. Similarly for $[J, J]\left(X^{*}, \xi_{2}\right)=0$.

4. For $A_{1}=X^{*}$ and $A_{2}=\xi_{3}$ we find:

$$
\begin{aligned}
{[J, J]\left(X^{*}, \xi_{3}\right) } & =\left[X^{*}, \xi_{3}\right]+J\left[J X^{*}, \xi_{3}\right]+J\left[X^{*}, J \xi_{3}\right]-\left[J X^{*}, J \xi_{3}\right]= \\
& =J\left[X^{*}, \xi^{*}\right]-\left[(\varphi X)^{*}, \xi^{*}\right],
\end{aligned}
$$

the brackets with $\xi_{3}$ being zero by (4.5). The horizontal component of the remaining two brackets is $\left([\varphi[X, \xi]-[\varphi X, \xi])^{* \prime}-d \hat{\eta}_{3}\left(X^{*}, \xi^{*}\right) \xi_{i}\right.$. The $\xi^{*}$ component as well as the vertical component vanish by assumption 2 ) in the statement. Finally, using Sasakian identities and the normality condition on $B$ we have $\left([\varphi[X, \xi]-[\varphi X, \xi])^{* \prime}=0\right.$.

5. Immediate computation shows that in the remaining "mixed" case $[J, J]\left(\xi_{i}, \xi^{*}\right)=0$.

6. We are left with the computation of $[J, J]$ on vertical fields. Obviously $[J, J]\left(\xi_{1}, \xi_{2}\right)=0$. Then

$$
\begin{aligned}
{[J, J]\left(\xi_{1}, \xi_{3}\right) } & =\left[\xi_{1}, \xi_{3}\right]+J\left[J \xi_{1}, \xi_{3}\right]+J\left[\xi_{1}, J \xi_{3}\right]-\left[J \xi_{1}, J \xi_{3}\right] \\
& =\left[\xi_{1}, \xi 3\right]+J\left[\xi_{2}, \xi_{3}\right]+J\left[\xi_{1}, \xi^{*}\right]-\left[\xi_{2}, \xi^{*}\right]=0
\end{aligned}
$$

by $\left[\xi, \xi_{j}\right]=2 \varepsilon_{i j k} \xi_{k}$ and (4.5). Same arguments show that $[J, J]\left(\xi_{2}, \xi_{3}\right)=$ 0 , thus completing the proof.

Remark 4.4. The Kähler form of $(P, g, J)$ is

$$
\omega=d \pi^{*} \eta+\pi^{*} \eta \wedge \eta_{3}-d \eta_{3} ;
$$

this shows that $d \omega \neq 0$, hence the structure is not Kählerian. A similar computation proves that $L_{\xi^{*}} J=L_{\xi_{3}} J=0$, thus $\xi^{*}$ and $\xi_{3}$ are infinitesimal automorphisms of the constructed complex structure.

Remark 4.5. The complex structure $J$ on $P$ depends on the choice of a 3-Sasakian structure on the fibre. But one can see that different choices of 3-Sasakian triples $\left\{\xi_{1}, \xi_{2}, \xi_{3}\right\}$ produce complex structures that are conjugated in $\operatorname{End}(T P)$.

We can now go back to the Stiefel manifolds $V_{2}\left(\mathbb{C}^{n+1}\right)$ and $\widetilde{V}_{4}\left(\mathbb{R}^{n+1}\right)$, and complete the proof of Theorems 3.1 (iii) and 3.2 (ii). We have just to verify that the induced Hopf bundles $V_{2}\left(\mathbb{C}^{n+1}\right) \rightarrow \mu^{-1}(0)$ and $\widetilde{V}_{4}\left(\mathbb{R}^{n+1}\right) \rightarrow \nu^{-1}(0)$ inherit from the inclusions $\nu^{-1}(0) \subset \mu^{-1}(0) \subset \mathbb{H} P^{n}$ horizontal distributions $\mathcal{H}$ satisfying the curvature properties of Proposition 4.2. Now property 1) simply express that the $s p(1)$-connection given by $\mathcal{H}$ is part of a $u(2)$-connection in the bundles $V_{2}\left(\mathbb{C}^{n+1}\right) \rightarrow$ $G r_{2}\left(\mathbb{C}^{n+1}\right)$ and $\widetilde{V}_{4}\left(\mathbb{R}^{n+1}\right) \rightarrow Z_{\widetilde{G} r_{4}\left(\mathbb{R}^{n+1}\right)}$, a fact easily recognized as in the case of hypercomplex structures in $V_{2}\left(\mathbb{C}^{n+1}\right)$ (cf. [14], proof of Thm 
1.10, as well as 15], Thm. 1.13). The meaning of property 2) is that the curvature of such a $s p(1)$-connection is given by a $(1,1)$-form with respect to the almost complex structure $J$. This follows for example from the computation carried out in [34], p. 63, for the Hopf bundle. One has to take into account that the rôle of the $U(1)$ and of the $S p(1)$-actions on both Stiefel manifolds correspond to the standard basic choice of left and right multiplication by scalars on $\mathbb{H}^{n+1}$. Then both on $V_{2}\left(\mathbb{C}^{n+1}\right)$ and on $\widetilde{V}_{4}\left(\mathbb{R}^{n+1}\right)$ the almost complex structure $J$ satisfies the compatibility conditions with the Sasakian structures of $\mu^{-1}(0)$ and $\nu^{-1}(0)$, as expressed by conditions 1) and 2) of Proposition 4.2. Note that the complex structure obtained in this way on $V_{2}\left(\mathbb{C}^{n+1}\right)$ projects to the complex structure underlying the Kähler structure of $G r_{2}\left(\mathbb{C}^{n+1}\right)$. This latter is well known to be not compatible with the quaternion Kähler structure of this Grassmannian. But it is precisely this quaternion Kähler structure which is lifted to a 3-Sasakian structure and then, by means of an appropriate circle bundle, to a hypercomplex structure on $V_{2}\left(\mathbb{C}^{n+1}\right)$, cf. [2], [14]. Hence our complex structure is not compatible with the standard hypercomplex one of $V_{2}\left(\mathbb{C}^{n+1}\right)$. This completes the proof of Theorem 3.1 (iii) and 3.2 (ii).

Proof of Corollary 3.1. It remains only to show that the total space of an induced $S^{1}$ Hopf bundle is minimal in $\left(S^{2 N-1}\right.$, can $)$. In fact, more generally, in a commutative diagram

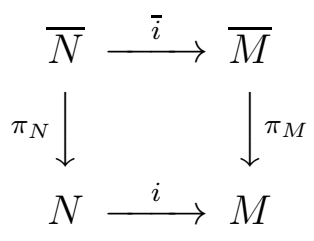

of immersions $i, \bar{i}$ and Riemannian submersions $\pi_{N}, \pi_{M}$ with totally geodesic fibres, we see that $N$ is minimal in $M$ if and only if $\bar{N}$ is minimal in $\bar{M}$. This follows by a direct computation of the mean curvature vector fields of $i$ and $\bar{i}$ using the Gauss formula of a submanifold and formula $(9.25$ a) in [6].

\section{Further Observations}

As mentioned in the introduction, the zero level set $\mu^{-1}(0) \subset \mathbb{H} P^{2}$ is diffeomorphic to a sphere $S^{5}$ and the projection to the reduced manifold can be identified with the Hopf fibration $S^{5} \rightarrow \mathbb{C} P^{2}$. Going to the next case $n=3$, the projection $\mu^{-1}(0) \rightarrow G r_{2}\left(\mathbb{C}^{4}\right)$, now an induced Hopf fibration, can be described by looking at the families of submanifolds in $G_{2}\left(\mathbb{C}^{4}\right)$ that are either Kähler-Einstein or quaternion 
Kähler. The Grassmannian $G r_{2}\left(\mathbb{C}^{4}\right)$ admits in fact a natural complex Kähler structure, as well as two distinct quaternion Kähler structures induced via the isomorphism of vector bundles $T G r_{2}\left(\mathbb{C}^{4}\right) \cong V \otimes V^{\perp}$ from the (almost) hypercomplex structure on the tautological vector bundle $V$ or on its orthogonal complement $V^{\perp}$ (cf. [27]). The families of submanifolds we want to look at on $G r_{2}\left(\mathbb{C}^{4}\right)$ are described as follows (see [7], [8], [26], [35]).

There are two families $\mathcal{F}, \mathcal{F}^{\prime}$ of complex projective planes $\mathbf{C} P^{2}$, $\mathrm{C} P^{2^{\prime}}$, a family $\mathcal{F}^{\prime \prime}$ of products $\mathbb{C} P^{1} \times \mathbb{C} P^{1}$, and a family $\mathcal{F}^{\prime \prime \prime}$ of spheres $S^{4}$; they are given by:

$$
\begin{aligned}
\mathcal{F}= & \left\{\text { planes contained in a } 3 \text {-space of } \mathbb{C}^{4}\right\}, \\
\mathcal{F}^{\prime}= & \left\{\text { planes through a line of } \mathbb{C}^{4}\right\}, \\
\mathcal{F}^{\prime \prime}= & \{\text { planes that are invariant for a hypercomplex } \\
& \left.\quad \text { structure } J \text { of } \mathbb{C}^{4}\right\}, \\
\mathcal{F}^{\prime \prime \prime}= & \{\text { planes given by pairs of lines in two fixed orthogonal } \\
& \left.\quad \text { planes of } \mathbb{C}^{4}\right\} .
\end{aligned}
$$

All these 4-dimensional submanifolds of $G r_{2}\left(\mathbb{C}^{4}\right)$ have nice intersection properties, some of which can also be formulated in terms of projective geometry of the lines in the 3 -dimensional space $\mathbb{C} P^{3}$, the context where the Klein quadric $\mathbb{C} Q^{4} \subset \mathbb{C} P^{5}$, isometric to $G r_{2}\left(\mathbb{C}^{4}\right)$, was first introduced. Instead of listing these intersection properties on $G r_{2}\left(\mathbb{C}^{4}\right)$ (cf. [26], pp. 508-512, for some of them; the remaining ones can be similarly deduced), we formulate the corresponding properties for the families of 5-dimensional submanifolds obtained at the Sasakian Einstein level as induced Hopf bundles over the members of families $\mathcal{F}, \ldots, \mathcal{F}^{\prime \prime \prime}$.

Proposition 5.1. The 9-dimensional Sasakian Einstein focal set

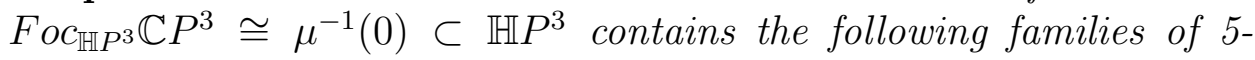
dimensional submanifolds, each of which fibers in circles over a complex or a quaternionic submanifold of $G r_{2}\left(\mathbb{C}^{4}\right)$. There are two families $\mathcal{E}, \mathcal{E}^{\prime}$ of Sasakian 5-spheres $S^{5}, S^{\prime 5}$, a family $\mathcal{E}^{\prime \prime}$ of Sasakian products $S^{3} \times S^{2}$ and a family $\mathcal{E}^{\prime \prime \prime}$ of products $S^{4} \times S^{1}$. The induced metrics on members of the families $\mathcal{E}, \mathcal{E}^{\prime}, \mathcal{E}^{\prime \prime}$ are Sasakian $\eta$-Einstein, and can be modified to Sasakian Einstein metrics by formula (2.1). The intersection properties of these 5-dimentional submanifolds are the following.

(a) Pairs of 5-spheres in the same family intersect in a circle, and pairs of 5-spheres in different families either do not intersect or intersect in an $S^{3}$. 
(b) Pairs of submanifolds of type $S^{3} \times S^{2}$ intersect either in an $S^{3}$ or in a pair of disjoint circles. A submanifold of type $S^{3} \times S^{2}$ intersects a 5-sphere in an $S^{3}$.

(c) Any submanifold of type $S^{4} \times S^{1}$ intersects a 5-sphere in a circle, intersects an $S^{3} \times S^{2}$ in two disjoint circles, and any pair of submanifolds of type $S^{4} \times S^{1}$ intersect in two disjoint circles.

This kind of geometry of submanifolds, now described for the level set $\mu^{-1}(0) \subset \mathbb{H} P^{3}$, can be formulated for all the zero level sets of moment maps appearing in the diagram of Corollary 3.1. There is such a level set for each odd dimension. For example, $\nu^{-1}(0) \subset \mathbb{H} P^{4}$ is diffeomorphic to a sphere $S^{7}$, yielding as reduced manifold $\mathbb{H} P^{1}$ and fibering in circles over its twistor space $\mathbb{C} P^{3}$, a Kähler submanifold of the Grassmannian $G r_{2}\left(\mathbb{C}^{5}\right)$. Thus Sasakian $(\eta)$-Einstein submanifolds of type $S^{5}$ and $S^{3} \times S^{2}$ can be determined in $S^{7}$ (cf. [31]), and intersection properties like in Proposition 5.1 are obtained.

The 11-dimensional example is the level set $\nu^{-1}(0) \subset \mathbb{H} P^{5}$, identified in Theorem 3.2 with an intersection of three focal sets in $\mathbb{H} P^{5}$. This 11dimensional manifold is diffeomorphic to the (unique) 3-Sasakian homogeneous manifold projecting in $S O(3)$ over the Wolf space $\widetilde{G} r_{4}\left(\mathbb{R}^{6}\right)$, and this latter manifold is isometric to $G r_{2}\left(\mathbb{C}^{4}\right)$. Thus again the geometry of 3-Sasakian and of Sasakian $(\eta)$-Einstein submanifolds $\nu^{-1}(0) \subset$ $\mathbb{H} P^{4}$ is obtained from the same starting point as Proposition 5.1.

Besides this kind of geometry of submanifolds, the Sasakian-Einstein level sets $\mu^{-1}(0)$ share with some of the 3 -Sasakian level sets $\nu^{-1}(0)$ a common expression of their Poincaré polynomials. We have in fact:

Proposition 5.2. The Poincaré polynomial of $\mu^{-1}(0) \subset \mathbb{H} P^{n}$ is given by:

$$
\operatorname{Poin}_{\mu^{-1}(0)}(t)=\sum_{i=0}^{\left[\frac{n-1}{2}\right]}\left(t^{4 i}+t^{4 n-3-4 i}\right)
$$

This is obtained by the Gysin sequence of the $S^{1}$-bundle $\mu^{-1}(0) \rightarrow$ $G r_{2}\left(\mathbb{C}^{n+1}\right)$, where the connecting homomorphism

$$
H^{p}\left(G r_{2}\left(\mathbb{C}^{n+1}\right)\right) \rightarrow H^{p+2}\left(G r_{2}\left(\mathbb{C}^{n+1}\right)\right)
$$

is given by the wedge product with the Kähler form of the Grassmannian. Since this wedge product is injective up to the middle real dimension $p+2=2 n-2$ (cf. [22], lemma 3.1, for the similar 3Sasakian situation), the Gysin sequence reduces to a series of short exact sequences finishing with

$$
H^{2 n-4}\left(G r_{2}\left(\mathbb{C}^{n+1}\right)\right) \rightarrow H^{2 n-2}\left(G r_{2}\left(\mathbb{C}^{n+1}\right)\right) \rightarrow H^{2 n-2}\left(\mu^{-1}(0)\right) .
$$


This allows to compute the Betti numbers of $\left(\mu^{-1}(0)\right)$ by differences of consecutive even Betti numbers in $G r_{2}\left(\mathbb{C}^{n+1}\right)$. The Poincaré polynomial of $G r_{2}\left(\mathbb{C}^{n+1}\right)$ is well known (see, for example, [9], p. 292) and by writing it as:

$$
\operatorname{Poin}_{G r_{2}\left(\mathbb{C}^{n+1}\right)}=\left(1+t^{2}+\ldots+t^{2 n-2}\right)\left(1+t^{4}+t^{4 m-4}\right),(n+1=2 m)
$$

$$
\operatorname{Poin}_{G r_{2}\left(\mathbb{C}^{n+1}\right)}=\left(1+t^{4}+\ldots+t^{4 m-4}\right)\left(1+t^{2}+t^{2 n}\right),(n+1=2 m+1)
$$

the conclusion is easily obtained.

The following table gives the Poincaré polynomial of $\mu^{-1}(0) \subset \mathbb{H} P^{n}$ for low values of $n$

\begin{tabular}{|l|c|}
\hline$n=3$ & $1+t^{4}+t^{5}+t^{9}$ \\
$n=4$ & $1+t^{4}+t^{9}+t^{13}$ \\
$n=5$ & $1+t^{4}+t^{8}+t^{9}+t^{13}+t^{17}$ \\
$n=6$ & $1+t^{4}+t^{8}+t^{13}+t^{17}+t^{21}$ \\
$n=7$ & $1+t^{4}+t^{8}+t^{12}+t^{13}+t^{17}+t^{21}+t^{25}$ \\
$n=8$ & $1+t^{4}+t^{8}+t^{12}+t^{17}+t^{21}+t^{25}+t^{29}$ \\
\hline
\end{tabular}

The Poincaré polynomial of $\mu^{-1}(0)$ can be compared with that of $\nu^{-1}(0)$, computed as for the homogeneous 3-Sasakian manifold $S O(n+$ $1) /(S O(n-3) \times S p(1))$. The latter has two different expressions, according to whether $n+1$ is even or odd (see [22] or [11], p. 28). For odd values of $n+1=2 k+3$ this expression is:

$$
\operatorname{Poin}_{\nu^{-1}(0)}(t)=\sum_{i=0}^{k-1}\left(t^{4 i}+t^{8 k-1-4 i}\right),
$$

and taking account of the dimensions, this is the same formula given in Proposition 5.2 for $\operatorname{Poin}_{\mu^{-1}(0)}(t)$. 


\section{REFERENCES}

[1] F. Battaglia, Applicazioni momento in geometria differenziale, Tesi di Dottorato, Univ. di Firenze (1993).

[2] F. Battaglia, A hypercomplex Stiefel manifold, Diff. Geom. and Appl. 6 (1996), 121-128.

[3] F. Battaglia, $S^{1}$-actions on quaternionic-Kähler manifolds, Proc. Amer. Math. Soc. 124 (1996), 2185-2192.

[4] F. Battaglia, Circle actions and Morse theory on quaternionic-Kähler manifolds, J. London Math. Soc. 59 (1999), 345-358.

[5] H. Baum, T. Friedrich, R. Grunewald, I. Kath, Twistors and Killing spinors on Riemannian manifolds, Teubner Verlagsgesellschaft, (1991).

[6] A. Besse, Einstein manifolds, Springer-Verlag (1987).

[7] J. Berndt, A new approach to the complex Grassmann manifolds $\mathbb{C} G_{2, m}$, Habilitationsschrift, Univ. Köln (1994).

[8] J. Berndt, Riemannian geometry of complex two-plane Grassmannians, Rend. Sem. Mat. Univ. Politec. Torino 55 (1997), 19-83.

[9] R. Bott, L. W. Tu, Differential Forms in Algebraic Topology, Springer-Verlag (1982).

[10] Ch. P. Boyer, K. Galicki, The twistor space of 3-Sasakian manifolds, Int. J. Math., 8 (1997), 31-60.

[11] Ch. P. Boyer, K. Galicki, 3-Sasakian manifolds, Essays on Einstein Manifolds, (C. LeBrun and M. Wang Eds), Surveys in Differential Geometry, vol. V, Int. Press (2000). available in http://xxx.lanl.gov/, hep-th 9810250.

[12] Ch. P. Boyer, K. Galicki, On Sasakian-Einstein Geometry, preprint, available in http://xxx.lanl.gov/, math.DG 9811098.

[13] Ch. P. Boyer, K. Galicki, B. Mann, The geometry and topology of 3-Sasakian manifolds, J. Reine Angew. Math., 455 (1994), 183-220.

[14] Ch. P. Boyer, K. Galicki, B. Mann, Hypercomplex structures on Stiefel manifolds, Ann. Global Anal. Geom. 14 (1996), 81-105.

[15] Ch. P. Boyer, K. Galicki, B. Mann, Hypercomplex structures from 3-Sasakian structures, J. Reine Angew. Math., 501 (1998), 81-105.

[16] T. E. Cecil, P. J. Ryan, Focal sets of submanifolds, Pac. J. Math., 78 (1978), 27-39.

[17] T. E. Cecil, P. J. Ryan, Focal sets and real hypersurfaces in complex projective space, Trans. Am. Math. Soc. 269 (1982), 481-499.

[18] J. Cheeger, D. E. Ebin, Comparison Theorems in Riemannian geometry, NorthHolland (1975).

[19] M. P. do Carmo, Riemannian Geometry, Birkhäuser (1992).

[20] K. Galicki, A generalization of the momentum mapping construction for quaternionic Kähler manifolds Commun. Math. Phys. 108 (1987), 117-138.

[21] K. Galicki, H. B. Lawson, Quaternionic reduction and quaternionic orbifolds, Math. Ann. 282 (1988), 1-21.

[22] K. Galicki, S.M. Salamon, Betti numbers of 3-Sasakian manifolds, Geom. Dedicata, 63 (1996), 45-68.

[23] R. Herrera, S. Salamon, Moduli and twistor spaces, Rend. di Mat. 17 (1997), 697-712.

[24] D. Joyce, Compact hypercomplex and quaternionic manifolds, J. Diff. Geom. 35 (1992), 743-761. 
[25] P. Z. Kobak, Quaternionic geometry and harmonic maps, $\mathrm{Ph}$. D. Thesis, Oxford (1993).

[26] S. Marchiafava, Su alcune sottovarietà che ha interesse considerare in una varietà kähleriana quaternionale, Rend. Mat. Appl., 10 (1990), 493-529.

[27] F.Morgan, Least-volume representatives of homology classes in $G\left(2, \mathbb{C}^{4}\right)$, Ann. Ecole Norm. Sup. 22 (1989), 127-135.

[28] B. O'Neill, Semi-Riemannian geometry, Academic Press (1983).

[29] L. Ornea, P. Piccinni, Locally conformal Kähler structures in quaternionic geometry, Trans. Am. Math. Soc. 349 (1997), 641-655.

[30] L. Ornea, P. Piccinni, Compact hyperhermitian-Weyl and quaternion Hermitian-Weyl manifolds, Ann. Global Anal. Geom. 16 (1998), 383-398.

[31] L. Ornea, P. Piccinni, Induced Hopf bundles and Einstein metrics, in "New developments in differential geometry, Budapest 1996", Kluwer Publ. (1998), 295-306.

[32] L. Ornea, P. Piccinni, Complex structures on some Stiefel manifolds, Bull. Soc. Sc. Math. Roumanie, Volume in memory of G. Vrănceanu, (2000), to appear.

[33] T. Otsuki, On focal elements and the spheres, Tôhoku Math. J. 17 (1965), 285-304.

[34] P. Piccinni, Quaternionic differential forms and symplectic Pontrjagin classes, Ann. di Mat. Pura e Appl. 129 (1981), 57-68.

[35] P. Piccinni, The Geometry of Positive Locally Quaternion Kähler Manifolds, Ann. Global Anal. Geom. 16 (1998), 255-272.

[36] M. Pontecorvo, Complex structures on quaternionic manifolds, Diff. Geom. Appl. 4 (1994), 163-177.

[37] S. Salamon, Quaternionic Kähler manifolds, Invent. Math. 67 (1982), 143-171.

[38] S. Tanno, Geodesic flows on $C_{L}$-manifolds and Einstein metrics on $S^{3} \times S^{2}$, in Minimal submanifolds and geodesics, Kaigai Publ., Tokyo (1978), 283-292.

[39] R. S. Ward, R. O. Wells jr., Twistor geometry and field theory, Cambridge monographs on mathematical physics (1990).

[40] J. A. Wolf, Spaces of constant curvature, Mc Graw Hill, (1967).

University of Bucharest, Faculty of Mathematics, 14 Academiei STR., 70109 BUCHAREST, ROMANIA

E-mail address: lornea@imar.ro

Università degli Studi di Roma "La Sapienza", Piazzale Aldo Moro 2, I-00185 Roma, ItALiA

E-mail address: piccinni@mat.uniroma1.it 